\title{
An empirical, yet practical way to predict the band gap in solids by using density functional band structure calculations
}

\author{
Ángel Morales-García, Rosendo Valero, Francesc Illas* \\ Departament de Ciència de Materials i Química Física \& Institut de Química Teòrica i \\ Computational (IQTCUB), Universitat de Barcelona, \\ c/ Martí i Franqués 1, 08028 Barcelona, Spain.
}

\begin{abstract}
Band structure calculations based on Density Functional Theory (DFT) with local or gradient corrected exchange-correlation potentials are known to severely underestimate the band gap of semiconducting and insulating materials. Alternative approaches have been proposed; from semiempirical setups, such as the so-called $\mathrm{DFT}+U$, to hybrid density functionals using a fraction of non-local Fock exchange, to modifications of semilocal density functionals. However, the resulting methods appear to be material dependent and lack theoretical rigor. The rigorous many-body perturbation theory based on $G W$ methods provides accurate results but at a very high computational cost. Hereby, we show that a linear correlation between the electronic band gaps obtained from standard DFT and $G W$ approaches exists for most materials and argue that $i$ ) this is a strong indication that the problem of predicting band gaps from standard DFT calculation arises from the assignment of a physical meaning to the Kohn-Sham energy levels rather than from intrinsic errors of the DFT methods and ii) it provides a practical way to obtain $G W$-like quality results from standard DFT calculations. The latter will be especially useful for systems where the unit cell involves a large number of atoms as in the case of doped or defect containing materials for which $G W$ calculations become unfeasible.
\end{abstract}

*corresponding author: francesc.illas@ub.edu 


\section{Introduction}

The Kohn-Sham (KS) implementation of density functional theory (DFT) formalism is widely applied to understand and predict the structural, electronic, magnetic and other properties of a myriad of molecules and of condensed matter systems. The KS equation yields, in principle, the exact density and the total energy of the system in the ground state. ${ }^{1,2}$ However, at variance with Hartree-Fock where Koopmans' theorem provides a well-defined meaning to the orbital energies, the eigenvalues of the KS equation are taken as single-particle excitation energy without fundamental physical basis. ${ }^{3,4}$ In fact, relying on the KS energy levels (or bands), either using the local density approximation (LDA) or generalized gradient approximation (GGA) functionals, results in a consistent 30 to $100 \%$ underestimation of the electronic band gap ( $\left.E_{\text {gap }}\right)$ of semiconducting compounds and insulators. ${ }^{5}$ This behavior appears to be independent of the nature of the system $^{6}$ and the underestimation of $E_{\text {gap }}$ has been attributed to their inherent lack of derivative discontinuity ${ }^{7,8}$ and delocalization error. ${ }^{9}$ It is clear, therefore, that accurate prediction of band gaps is one of the critical challenges in DFT with potentially wide applications in several research fields such as photocatalytic processes. ${ }^{10}$

In the case of finite systems, the problem of the electronic band gap can be circumvented by relying on total energy differences, i.e. through the $\triangle \mathrm{SCF}$ approach. ${ }^{11}$ This approach reproduces successfully the experimental band gaps for most of finite systems. ${ }^{12}$ The $\Delta \mathrm{SCF}$ involves taking differences of total energy of a given $N$ electron system and that of the system with $N+1$ or $N-1$ electronic system which, unless some artificial charge compensating scheme is used, becomes unfeasible in periodic calculations. To overcome the drawback of LDA and GGA on estimating band gaps semiempirical approaches have been proposed such as DFT $+U$ which, unfortunately, introduces a parameter external to the theory. It has been proposed that $U$ can be obtained directly from constrained DFT calculations, and it can also be tuned to resemble the experimental band gap. ${ }^{13}$ For different solid state materials, however, $U$ has different values and must be chosen for each system separately. ${ }^{14,15}$ On the other hand, the hybrid density functionals, originally developed to improve the description of the ground state energetics of small molecules, ${ }^{16}$ have been shown to improve the description of $E_{\text {gap }}$ of semiconductor systems. ${ }^{17-20}$ These hybrid schemes contain an admixture of non-local Fock and/or additionally screen the interelectronic Coulomb potential in the exchange term. ${ }^{21}$ For instance, the so-called HSE06 functionalError! Bookmark not defined. applies a screened Coulomb potential only to the exchange interaction in order to screen the longrange part of the HF exchange. These parameters allow one to modulate both, the Fock contribution and the range separation, parameter for a given system to reproduce the experimental $E_{\text {gap. }}{ }^{22}$ Note that for $\mathrm{TiO}_{2}$, either rutile or anatase polymorphs, a $12.5 \%$ of Fock exchange is necessary to 
reproduce the band gap ${ }^{19}$ whereas for $\mathrm{ZnO}$ this is achieved ${ }^{20}$ with $25 \%$ of Fock exchange as in the well-known PBE0 functional and 35\% Fock exchange is needed to properly describe NiO. ${ }^{24} \mathrm{~A}$ similar study reports the performance of several exchange correlation functionals in predicting the band gap of a series of transition metal oxides and chalcogenides. ${ }^{25}$ Hence, hybrid functionals appear to be material dependent. These limitations are often attributed to the excited state intrinsic nature of $E_{\text {gap }}$ which, consequently, cannot be properly described from standard DFT calculations. ${ }^{26}$ Recently, the performance of the screened exchange (SX) constant functional on the GW100 set has been analyzed. ${ }^{27}$ Although an excellent agreement for the computed values of the ionization potential and optical band gaps with both $\operatorname{CCSD}(\mathrm{T})$ and experiment is found, the material dependence is clearly shown because the employed hybrid functionals are those so-called dielectric dependent hybrid (DDH) functionals ${ }^{28,29}$ where the amount of Fock exchange is chosen according to the dielectric constant — an external to theory property — of the material.

To a large extent, the failure of standard LDA or GGA calculations to reproduce the band gap comes from an inappropriate interpretation of the KS eigenvalues; a feature which is wellknown in solid state physics and materials science and has been discussed very recently in the framework of the so-called Generalized Kohn-Sham (GKS) theory. ${ }^{30}$ Yet, prediction of band gaps from KS band structure is widely accepted from a pragmatic point of view. ${ }^{31}$ Rigorously speaking, the representation of quasiparticles requires the use of many-body techniques such as the Many Body Perturbation Theory (MBPT) based on $G W$ methods as proposed long ago by Hedin ${ }^{32}$ to describe the energies of electronic excitation spectra of solids. Clearly, $G W$ emerges as the most physically grounded way to accurately predict $E_{\text {gap }}$ based on the quasiparticle energies despite its huge computational cost, especially in the self-consistent quasiparticle approach. ${ }^{33}$ The exceedingly large computational cost has led to simplified, yet rigorously grounded, approaches such as $G_{0} W_{0}$ where the Green's function $(G)$ and screened dielectric potential $(W)$ are computed from the KS density without further iterations. Nowadays, quasiparticle energies based on $G_{0} W_{0}$ calculations, ${ }^{34,35}$ on top of the DFT electron density obtained from standard exchange-correlation functionals (LDA and/or GGA), constitutes one of the most promising strategies to predict and reproduce the experimental band gaps theoretically in solids.

In addition to the $\mathrm{DFT}+U$, hybrid approaches and $G W$ methods mentioned above, other developments aimed at improving the accuracy of band gaps obtained from LDA or GGA calculations have relied on modifications to the density functionals. Thus, excited state corrections were employed for LDA to obtain band gaps for semiconductors in reasonable agreement with experimental evidences. ${ }^{36}$ A modification of the exchange potential proposed by Becke and Johnson ${ }^{37}$ was shown to agree well with experiment for several semiconductors and insulators, ${ }^{38}$ 
although further improvements were needed to account for systems with localized states. ${ }^{39}$ In a different study, a scaled modified LDA functional improved the band gap for atoms, molecules, and solids. ${ }^{40}$ All these studies employed the KS band gap as an approximation to the fundamental gap. However, generalized $\triangle \mathrm{SCF}$ calculations of the band gap of solids for LDA and GGA functionals reduce the KS band gap errors by about $70 \%{ }^{41}$ The latter work strongly suggests that the error in the band gap of solids is mostly due to the inappropriate interpretation of KS eigenvalues, as we stated above.

The quasiparticle energies are related to photoelectron spectroscopy, ${ }^{42}$ and are thus connected to the photoemission and its inverse processes with $(N-1)$ and $(N+1)$ electronic system, respectively. ${ }^{43}$ Thus, $E_{\text {gap }}$ calculated as $\triangle \mathrm{SCF}$ and $G_{0} W_{0}$ are comparable. ${ }^{44}$ Recently, machine learning techniques focusing on statistical indicators have been applied to create models that enable predicting the band gaps. ${ }^{45,46}$ In these studies, it is observed that the prediction of $E_{\text {gap }}$ through the KS levels arising from standard exchange-correlation functionals such as LDA or GGA seem to suffer from a rather systematic error. Additionally, a linear relationship between the band gap calculated as standard DFT (LDA/GGA) and as $G_{0} W_{0}$ has been reported for a large series of ZnO polymorphs. ${ }^{47}$ Based on these findings, hereby a feasible, practical yet accurate, empirical model to predict the experimental band gap from a standard DFT calculation is proposed. The empirical model reported here should be useful to predict $E_{\text {gap }}$ from a virtual screening of a large set of materials involving large unit cells for which the use of more accurate methods become prohibitive. It must be noted that our study follows the same argument reported recently by Baerends, ${ }^{48}$ where a method based on integer electron systems (an ensemble of $N$ - and $(N+1)$-electron systems) was proposed.

\section{Choice of a data set}

The empirical model reported in the present work emerges from the analysis of a test set of over 66 semiconducting and insulating materials ${ }^{49}$ with available experimental band gaps in the range of 0.1-9 eV. This database has been selected from data reported in the literature and hereby we focus on analyzing some emerging interesting and not so far described relationships. For the computational details corresponding to the calculated data of the selected solid semiconductors the interested reader is addressed to the set of references included in the Supporting Information. We note here that these have been selected among those reporting results from PBE and/or $G_{0} W_{0}$ calculations obtained from different codes. 


\section{Results and discussion}

Let us start by considering the overall accuracy of $G_{0} W_{0}$ band gaps to further argue that they can be taken as an appropriate reference. For the above mentioned 66 compound data set (Tables S1 and S2), Figure 1 shows a quantitatively enough linear correlation between experimental and $G_{0} W_{0}$ electronic band gap as in Eq. (1),

$$
E_{\text {gap }}(\exp )=0.998 \cdot E_{\text {gap }}\left(G_{0} W_{0}\right)+0.014
$$

with a regression coefficient of 0.97 and a small offset of $0.014 \mathrm{eV}$ only; a strong indication that calculated $E_{\text {gap }}\left(G_{0} W_{0}\right)$ values are reliable. Next, for the same dataset, Figure 2 reports the correlation between the $E_{\text {gap }}$ values obtained from the standard PBE functional $E_{\text {gap }}(\mathrm{PBE})$ and the $E_{\mathrm{gap}}\left(G_{0} W_{0}\right)$ values. A similar statistical analysis predicts a linear relationship as in Eq. (2),

$$
E_{\text {gap }}\left(G_{0} W_{0}\right)=1.358 \cdot E_{\text {gap }}(\mathrm{PBE})+0.904
$$

with a regression coefficient of 0.91 , still meaningful enough as discussed later on. Note, however, that this linear relationship involved a rather large systematic offset. For all these 66 materials, $E_{\text {gap }}(\mathrm{PBE})$ results are located above the "ideal" correlation indicating that the KS derived band gap values from this GGA type DFT method are systematically lower than their $G_{0} W_{0}$ counterparts, as expected. Hence, the deviations from experimental data reported in the Supporting Information confirm a systematic underestimate of the $E_{\mathrm{gap}}(\mathrm{PBE})$. The underestimate is dramatically reduced when considering $E_{\text {gap }}\left(G_{0} W_{0}\right)$ as already discussed (see Eq. (1), Tables S1, S2, and Figure S1). Figure 2 shows that most of the compounds in the database, especially the set of non oxide semiconductors, are in or near the regression line. However, deviations of about $2 \mathrm{eV}$, thus larger than the offset in Eq. (2), are observed in a few oxide compounds, e.g., $\mathrm{MnO}, \mathrm{ZnO}, \mathrm{CoO}, \mathrm{SnO}_{2}$, $\mathrm{CuAlO}_{2}$ and $\mathrm{CuInO}_{2}$ (highlighted inset Figure 2). For convenience, these have been omitted in the linear fitting although it is noted that $G_{0} W_{0}$ improves significantly their corresponding $E_{\text {gap }}$ (see Figure 1). Another set of compounds with $E_{\text {gap }}<1 \mathrm{eV}$ also exhibit deviations because in these cases GGA type DFT methods do not only fail to reproduce the experimental values but incorrectly describe them as metals with $E_{\text {gap }}$ values close to zero (see Tables S1 and S2 for further details).

We now discuss two different error measures in Table 1, the mean absolute error (MAE) and the root-mean-square error (RMSE). Both are some of the most widely used error measures in quantum chemistry. $G_{0} W_{0}$ method predicts successfully band gaps closer to experiment while PBE has the worst error measures for both non oxide and oxide compounds on the basis of MAE and RMSE parameters, see Table 1. However, it must be noted that MAE and RMSE show high values for oxide compounds indicating a worse correlation compared to non oxide compounds. This is consistent with the good correlation in Figure 1 and confirms that $G W$ methods, even at the simplest 
$G_{0} W_{0}$ level, provide a rather accurate estimate of the electronic band gap in solids. However, the high computational cost severely limits its use to materials with relative small unit cell making unfeasible the calculation in doped or non-stoichiometric systems where large unit cells are required. Nevertheless, the reasonably good linear fitting in Eq. (2) and Figure 2 allows one to predict a $G_{0} W_{0}$ quality electronic band gap, and hence to obtain a reliable prediction of the experimental value, from a simple standard DFT calculation with the PBE functional, provided PBE is able to at least to qualitatively describe the semiconducting or insulating character of the system of interest.

To confirm that such practical model is appropriate, a set of materials (Table S3), not included in the dataset, is chosen to estimate the $G_{0} W_{0}$ electronic band gap from PBE calculations. The thus estimated $E_{\text {gap }}\left(G_{0} W_{0}\right)$ value is then compared with $E_{\text {gap }}(\exp )$ using Eq. (1). The predictive capability of the present approach is clearly shown in Figure 3. For a set of 13 materials, the prediction of $E_{\text {gap }}(\exp )$ from Eqs. (1) and (2) compares well with experiment thus validating the approach and providing a new practical method to predict the electronic band gap in solids.

\section{Conclusions}

In summary, results in the present work show that, to a large extent, the failure of DFT predicted band gaps arises from the broadly used but not rigorously grounded interpretation of the KS levels. Moreover, the analysis of a rather large dataset of calculated and experimental results shows a good linear correlation between experimental and calculated $G_{0} W_{0}$ values and between the latter and values from standard DFT calculations using a GGA type functional, thus opening the way to reliable predictions for materials where $G_{0} W_{0}$ calculations are unfeasible with the only condition that PBE does not lead to a vanishing gap. Finally, it is tempting to relate the success of $G_{0} W_{0}$ on top of the PBE electron density to predict band gaps of semiconductors and insulators to the claim of Medevev et al. ${ }^{50}$ that, unless relying of GKS, ${ }^{23}$ global or screened hybrid functionals may provide better numerical estimates of some properties, such as the band gap, ${ }^{51}$ at the cost of using a density deviating more from the exact one. We point out that the present empirical model will be useful in the field of engineering and code development offering an accurate estimation of the electronic band gap of many solids with an affordable computational cost. 
Figure 1. Linear fitting of the experimental versus $G_{0} W_{0}$ calculated electronic band gap of 66 materials in the dataset (Tables $\mathrm{S} 1$ and $\mathrm{S} 2)$. The green line represents the straight line: $E_{\text {gap }}(\exp )=$ $0.998 \cdot E_{\text {gap }}\left(G_{0} W_{0}\right)+0.014$ with a linear correlation coefficient of 0.97 . The bottom figure corresponds to a zoom of the gray area marked inset of the top figure.
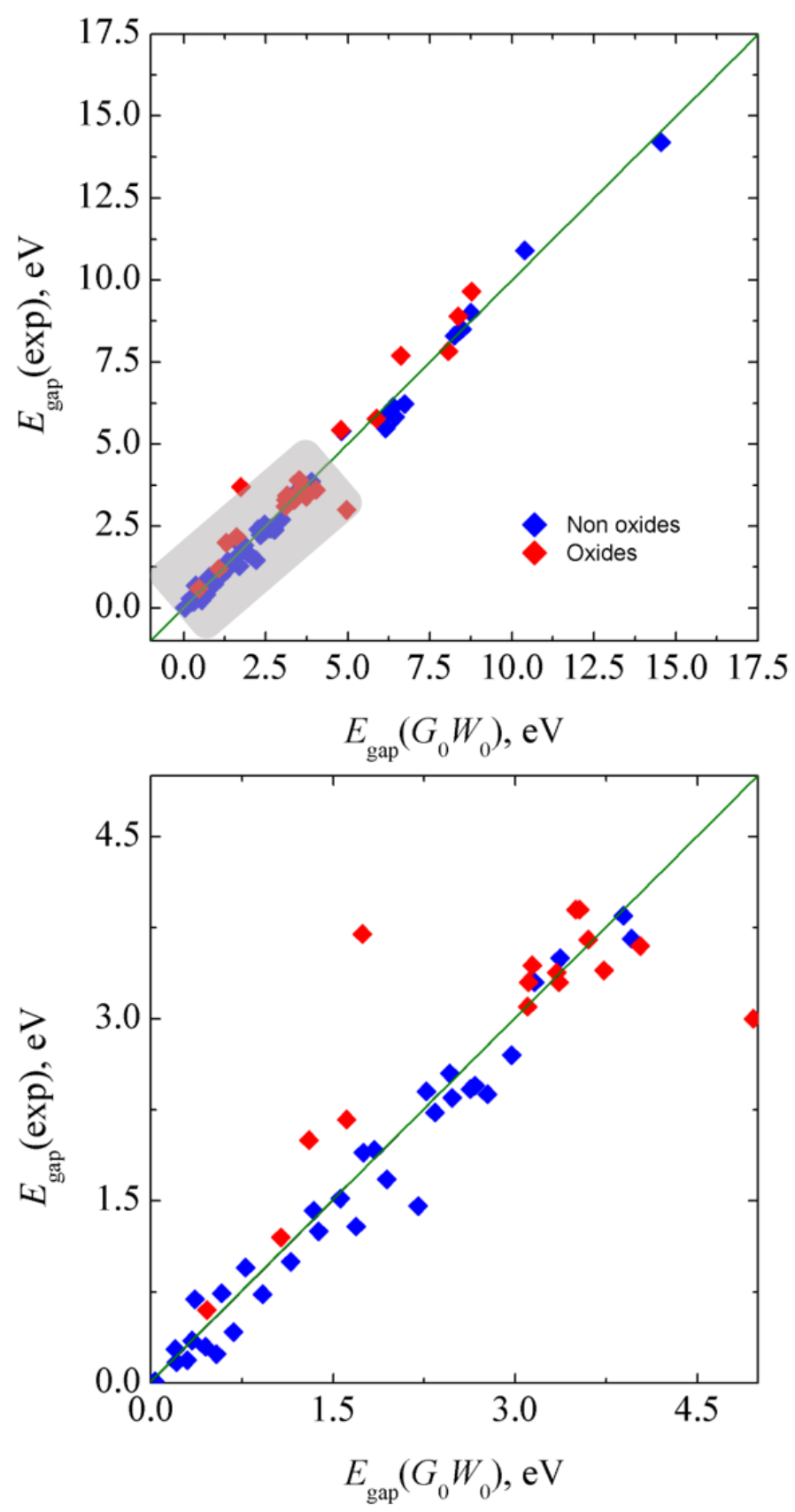
Figure 2. Linear fitting of the $G_{0} W_{0}$ versus DFT calculated electronic band gap (DFT) at PBE level of 66 materials in the dataset (Tables S1 and S2). The solid gray line represents a slope equal to 1 and the green line represents the straight line: $E_{\mathrm{gap}}\left(G_{0} W_{0}\right)=1.358 \cdot E_{\mathrm{gap}}(\mathrm{PBE})+0.904$ with a linear correlation coefficient of $0.91 . \mathrm{MnO}, \mathrm{ZnO}, \mathrm{CoO}, \mathrm{CuInO}_{2}, \mathrm{CuAlO}_{2}$ and $\mathrm{SnO}_{2}$ are not considered in the linear fitting.

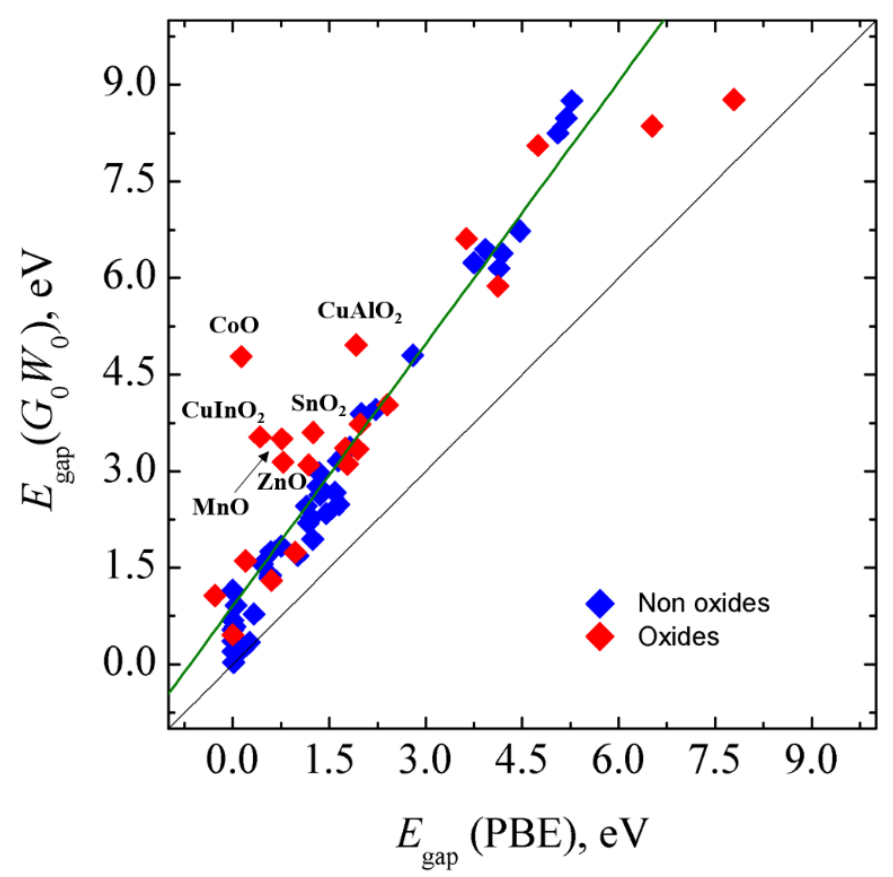


Figure 3. Predicted and experimental electronic band gap. The red line corresponds to the straight line of the predictive model, Eq. (1), and the green stars are the semiconductors considered to validate the model proposed. Further information can be found in Table S3.

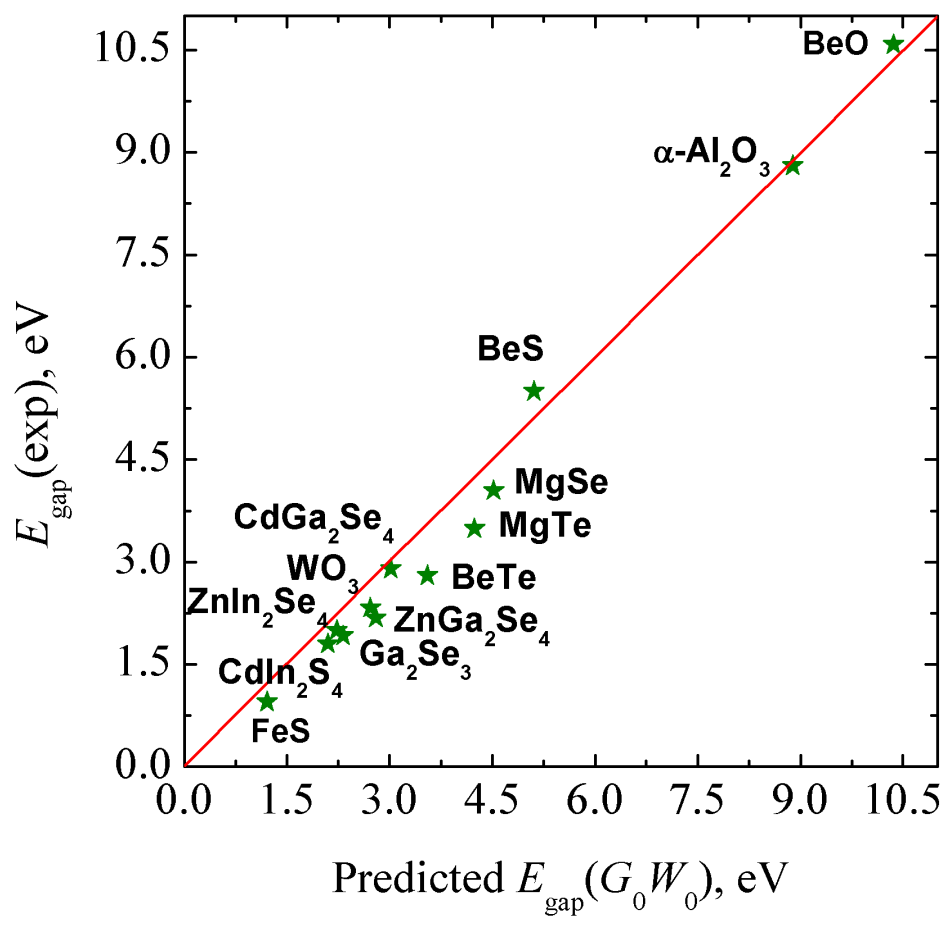


Table 1. Mean absolute error (MAE) and root-mean-square error (RMSE) of PBE and $G_{0} W_{0}$ calculated band gap of oxides and non oxides compounds (see Tables S1 and S2).

\begin{tabular}{lll}
\hline & PBE & $\boldsymbol{G}_{\mathbf{0}} \boldsymbol{W}_{\mathbf{0}}$ \\
\hline & Non Oxides & \\
MAE & 1.32 & 0.23 \\
RMSE & 1.76 & 0.29 \\
& \multicolumn{2}{c}{ Oxides } \\
MAE & 2.19 & 0.51 \\
RMSE & 2.45 & 0.74 \\
\hline
\end{tabular}




\section{ASSOCIATED CONTENT}

\section{Supporting Information}

The Supporting Information is available free of charge on the ACS Publications website at DOI:.......

Tables S1, S2 and S3 compile all the materials considered in this study, their space group and their electronic band gaps. Figure $\mathrm{S} 1$ shows $E_{\mathrm{gap}}\left(G_{0} W_{0}\right), E_{\mathrm{gap}}(\mathrm{PBE})$ and $E_{\text {gap }}(\exp )$ of all data reported in Tables S1 and S2.

\section{AUTHOR INFORMATION}

\section{Notes}

The authors declare no competing financial interests.

*corresponding Author: e-mail: francesc.illas@ub.edu

\section{ACKNOWLEDGMENT}

This research was supported by the Spanish MINECO/FEDER CTQ2015-64618-R grant and, in part, by Generalitat de Catalunya (grants 2014SGR97 and XRQTC) and by the NOMAD Center of Excellence project, which received funding from the European Union's Horizon 2020 research and innovation programme under grant agreement No 676580. A. M. G. thanks to Spanish Ministerio de Economía y Competitividad for the Juan de la Cierva postdoctoral grant (FJCI-2015-23760) and F. I. acknowledges additional support from the 2015 ICREA Academia Award for Excellence in University Research. Computational time at the Marenostrum supercomputer has been provided by the Barcelona Supercomputing Centre through grants from Red Española de Supercomputación and the COMPHOTOCAT project 2014112608 of the Partnership for Advanced Computing in Europe (PRACE). Valuable comments from Dr. Francesc Viñes are greatly appreciated. 


\section{REFERENCES}

(1) Hohenberg, P.; Kohn, W. Inhomogeneous Electron Gas. Phys. Rev. 1964, 136, B864-B871.

(2) Kohn, W.; Sham, L. J. Self-Consistent Equations Including Exchange and Correlation Effects. Phys. Rev. 1965, 140, A1133-A1138.

(3) Mermin, N. D. Thermal Properties of the Inhomogeneous Electron Gas. Phys. Rev. 1965, 137, A1441-A1443.

(4) Perdew, J. P.; Norman, M. R. Electron removal energies in Kohn-Sham density-functional theory. Phys. Rev. B 1982, 26, 5445-5450.

(5) Wang, C. S.; Perdew, J. P. Density-Functional Theory of Excitation Spectra of Semiconductors: Application to Si. Phys. Rev. Lett. 1983, 51, 597-600.

(6) Becke, A. D. Perspective: Fifty years of density-functional theory in chemical physics. J. Chem. Phys. 2014, 140, 18A301.

(7) Sham, L. J.; Schlüter, M. Density-Functional Theory of the Energy Gap. Phys. Rev. Lett. 1983, 51, 1888-1891.

(8) Mori-Sánchez, P.; Cohen, A. J. The Derivative Discontinuity of the Exchange-Correlation Functional. Phys. Chem. Chem. Phys. 2014, 16, 14378-14387.

(9) Mori-Sánchez, P.; Cohen, A. J.; Yang, W. Localization and Delocalization Errors in Density Functional Theory and Implications for Band-Gap Prediction. Phys. Rev. Lett. 2008, 100, 146401.

(10) Chang, X.; Wang, T.; Gong, J. $\mathrm{CO}_{2}$ photo-reduction: insights into $\mathrm{CO}_{2}$ activation and reaction on surfaces of photocatalysts. Energy Environ. Sci. 2016, 9, 2177-2196.

(11) Jones, R. O.; Gunnarsson, O. The density functional formalism, its applications and prospects. Rev. Mod. Phys. 1989, 61, 689-746.

(12) Cohen, A. J.; Mori-Sánchez, P.; Yang, W. Fractional charge perspective on the band gap in density-functional theory. Phys. Rev. B 2008, 77, 115123.

(13) Himmetoglu, B.; Floris, A.; de Gironcoli, S.; Cococcioni, M. Hubbard-Corrected DFT Energy Functionals: The LDA $+U$ Description of Correlated Systems. Int. J. Quantum Chem. 2014, $114,14-49$.

(14) Anasimov, V. I.; Zaanen, J.; Anderson, O. K. Band theory and Mott insulators: Hubbard $U$ instead of Stoner I. Phys. Rev. B 1991, 44, 943-954. 
(15) Verma, P.; Truhlar, D. G. Does DFT $+U$ Mimic Hybrid Density Functionals? Theor. Chem. Acc. 2016, 135, $182(1-15)$.

(16) Becke, A. D. Density-functional thermochemistry. III. The role of exact exchange. J. Chem. Phys. 1993, 98, 5648-5652.

(17) Muscat, J.; Wander, A.; Harrison, N. M. On the prediction of band gaps from hybrid functional theory. Chem. Phys. Lett. 2001, 342, 397-401.

(18) Chan, M. K. Y.; Ceder G. Efficient Band Gap Prediction for Solids. Phys. Rev. Lett. 2010, 105, 196403.

(19) Perdew J. P. et al. Understanding band gaps of solids in generalized Kohn-Sham theory. Proc. Natl. Acad. Sci. 2017, 114, 2801-2806.

(20) Tran, F.; Blaha, P. Importance of the Kinetic Energy Density for Band Gap Calculations in Solids with Density Functional Theory. J. Phys. Chem. A 2017, 121, 3318-3325.

(21) Heyd, J.; Scuseria, G. E. Hybrid functionals based on a screened Coulomb potential. J. Chem. Phys. 2003, 118, 8207.

(22) Ko, K. C.; Lamiel-García, O.; Lee, J. Y.; Illas, F. Performance of a modified hybrid functionals in the simultaneous description of stoichiometric and reduced $\mathrm{TiO}_{2}$ polymorphs. Phys. Chem. Chem. Phys. 2016, 18, 12357-12367.

(23) Viñes, F.; Illas, F. Electronic structure of stoichiometric and reduced $\mathrm{ZnO}$ from periodic relativistic all electron hybrid density functional calculations using numeric atom-centered orbitals. J. Comput. Chem. 2017, 38, 523-529.

(24) Moreira, I. de P.R.; Illas. F.; Martin, R. L. Effect of Fock exchange on the electronic structure and magnetic coupling in NiO. Phys. Rev. B, 2002, 65, 155102.

(25) Li, W.; Walther, C. F. J.; Kuc, A.; Heine, T. Density Functional Theory and Beyond for BandGap Screening: Performance for Transition-Metal Oxides and Dichalcogenides. J. Chem. Theory Comput. 2013, 9, 2950-2958.

(26) Godby, R. W.; Schlüter, M.; Sham, L. J. Quasiparticle energies in GaAs and AlAs. Phys. Rev. $B$ 1987, 35, 4170 .

(27) Braward, N. P.; Govoni, M.; Vörös, M.; Galli, G. Performance and Self-Consistency of the Generalized Dielectric Dependent Hybrid Functional. J. Chem. Theory Comput. 2017, 13, 3318-3325. 
(28) Skone, J. H.; Govoni, M.; Galli, G. Self-consistent hybrid functional for condensed systems. Phys. Rev. B: Condens. Matter Mater. Phys. 2014, 89, 195112.

(29) Gerosa, M.; Bottani, C. E.; Caramella, L.; Onida, G.; Di Valentin, C.; Pacchioni, G. Electronic structure and phase stability of oxide semiconductors: Performance of dielectric-dependent hybrid functional DFT, benchmarked against $G W$ band structure calculations and experiments. Phys. Rev. B: Condens. Matter Mater. Phys. 2015, 91, 155201.

(30) Perdew, J. P.; Yang, W.; Burke, K.; Yang, Z.; Gross. E. K. U.; Scheffler, M.; Scuseria, G. E. Henderson, T.M.; Zhang, I. Y.; Ruzsinszky, A.; Peng, H.; Sun, J.; Trushin, E.; Görling. A. Understanding band gaps of solids in generalized Kohn-Sham theory, Proc. Natl. Acad. Sci. 2017, 114, 2801-2806.

(31) Baerends, E. J.; Gritsenko, O. V.; van Meer, R. The Kohn-Sham Gap, The Fundamental Gap and The Optical Gap: The Physical Meaning of Occupied and Virtual Kohn-Sham Orbital Energies. Phys. Chem. Chem. Phys. 2013, 15, 16408-16425.

(32) Hedin, L. New Method for Calculating the One-Particle Green's Function with Application to the Electron-Gas Problem. Phys. Rev. 1965, 139, A796-A823.

(33) Rostgaard, C.; Jacobsen, K. W.; Thygesen, K. S. Fully self-consistent $G W$ calculations for molecules. Phys. Rev. B 2010, 81, 085103.

(34) Aulbur, W. G.; Städele, M.; Görling, A. Exact-exchange-based quasiparticle calculations. Phys. Rev. B 2000, 62, 7121.

(35) Fleszar, A. LDA, $G W$, and exact-exchange Kohn-Sham scheme calculations of the electronic structure of sp semiconductors. Phys. Rev. B 2001, 64, 245204.

(36) Remediakis, I. N.; Kaxiras, E. Band structure calculation for semiconductors within generalized-density-functional-theory. Phys. Rev. B 1999, 59, 5536.

(37) Becke, A. D.; Johnson, E. R. A simple effective potential for exchange. J. Chem. Phys. 2006, $124,221101$.

(38) Tran, F.; Blaha, P. Accurate band gaps of semiconductors and insulators with a semilocal exchange-correlation potential. Phys. Rev. Lett. 2009, 102, 226401.

(39) Jiang. H. Band gaps from the Tran-Blaha modified Becke-Johnson approach: a systematic investigation. J. Chem. Phys. 2013, 138, 134115.

(40) Zheng, X.; Cohen, A. J.; Mori-Sánchez, P.; Hu, X.; Yang. W. Improving band gap prediction in density functional theory from molecules to solids. Phys. Rev. Lett. 2011, 107, 026403. 
(41) Chan, M. K. Y.; Ceder, G. Efficient band gap prediction for solids. Phys. Rev. Lett. 2010, 105, 196403.

(42) Rinke, P.; Qteish, A.; Neugebauer, J.; Scheffler, M. Exciting prospects for solids: Exactexchange based functionals meet quasiparticle energy calculations. Phys. Stat. Sol. (B) 2008, $245,929-945$.

(43) Onida, G.; Reining, L.; Rubio A. Electronic excitations: density-functional versus many-body Green's-function approaches. Rev. Mod. Phys. 2002, 74, 601-659.

(44) Morales-García, A.; Valero, R.; Illas, F. Performance of the $G_{0} W_{0}$ Method in Predicting the Electronic Gap of $\mathrm{TiO}_{2}$ Nanoparticles. J. Chem. Theory Comput. 2017 Article ASAP (DOI: 10.1021/acs.jctc.7b00308).

(45) Lee, J.; Seko, A.; Shitara, K.; Nakayama, K.; Tanaka, I. Prediction model of band gap for inorganic compounds by combination of density functional theory calculations and machine learning techniques. Phys. Rev. B 2016, 93, 115104.

(46) Pilania, G.; Gubernatis, J. E.; Lookman, T. Multi-fidelity machine learning models for accurate bandgap predictions of solids. Comput. Mater. Sci. 2017, 129, 156-163.

(47) Demiroglu, I.; Tosoni, S.; Illas, F.; Bromley, S. T. Bandgap engineering through nanoporosity. Nanoscale 2014, 6, 1181-1187.

(48) Baerends, E. J. From the Kohn-Sham band gap to the fundamental gap in solids. An integer electron approach. Phys. Chem. Chem. Phys. 2017, 19, 15639-15656.

(49) Madelung, O. Semiconductors: Data Handbook (Springer-Verlag, New York, 2004), 3rd ed.

(50) Medvedev, M. G.; Bushmarinov, I. S.; Sun, J.; Perdew, J. P.; Lyssenko, K. A. Density functional theory is straying from the path toward the exact functional. Science 2017, 355, 4952.

(51) Garza, A. J.; Scuseria. G. E. Predicting Band Gaps with Hybrid Density Functionals. J. Phys. Chem. Lett. 2016, 7, 4165-4170. 
TOC GRAPHICS

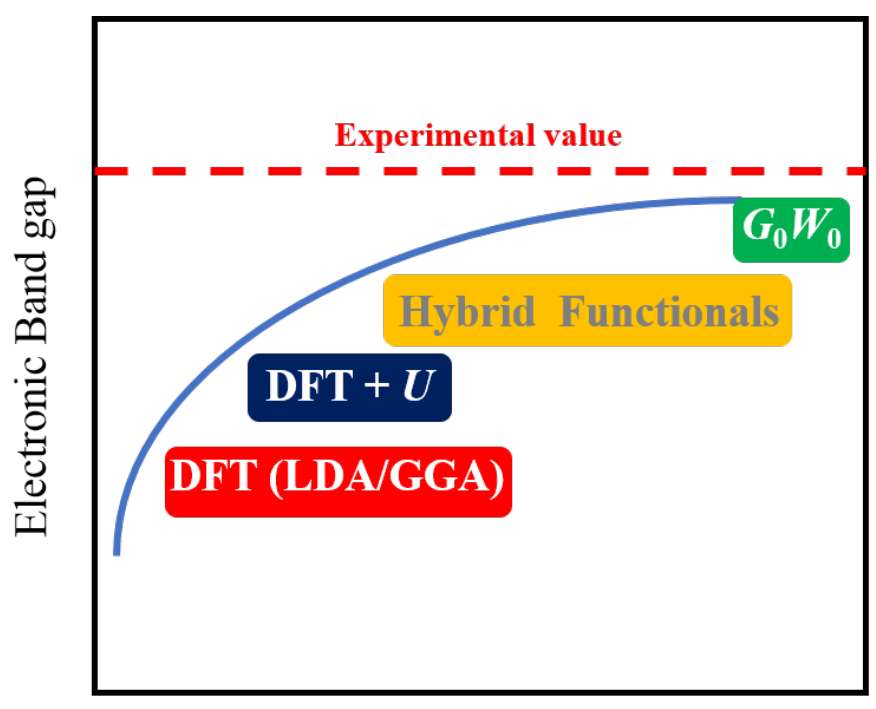

Level of calculation 